\title{
Nonsteroidal Anti-Inflammatory Drugs: A Potential Pharmacological Treatment for Intracranial Aneurysm
}

\author{
Courtney L. Fisher Stacie L. Demel \\ Department of Pharmacology and Toxicology, Michigan State University, East Lansing, MI, USA
}

Keywords

Aneurysm · Nonsteroidal anti-inflammatory drugs · Aspirin · Cyclooxygenase · Inflammation

\begin{abstract}
Background: Saccular intracranial aneurysms (IAs) are outpouchings of the vessel wall of intracranial arteries. Rupture of IAs results in subarachnoid hemorrhage which is associated with high morbidity and mortality. Surgical interventions, such as clipping and coiling, have associated risks. Currently, there are no proven pharmacological treatments to prevent the growth or rupture of IAs. Infiltration of proinflammatory cytokines in response to increased wall sheer stress is a hallmark of IA. Nonsteroidal anti-inflammatory drugs (NSAIDs) are being investigated as potential therapeutic agents for reduction in growth and/or prevention of IA through inhibition of inflammatory pathways. Summary: This review will discuss the role of NSAIDs in attenuating the inflammation that drives IA progression and rupture. There are two main subtypes of NSAIDs, nonselective COX and selective COX-2 inhibitors, both of which have merit in treating IA. Evidence will be presented which shows that NSAIDs inhibit several key inflammatory mediators involved in IA progression including nuclear factor- $\mathrm{kB}$, tumor necrosis factor- $\alpha$, and matrix metalloproteinases. In addition, the role of NSAIDs in limiting inflammatory cell adhesion to endothelial cells and attenuating endothelial cell senescence will be discussed. Key Messages: There is an abundance of basic science and preclinical data that support NSAIDs as a promising treatment for IA. Additionally, a combination treatment strategy of low-dose aspirin given concomitantly with a selective COX-2 inhibitor may result in a reduced side effect profile compared to aspirin or selective COX-2 inhibitor use alone. Several large clinical trials are currently planned to further investigate the efficacy of NSAIDs as an effective nonsurgical treatment for IAs.




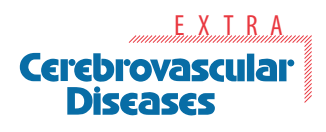

\begin{tabular}{l|l}
\hline Cerebrovasc Dis Extra 2019;9:31-45 \\
\hline DOI: 10.1159/000499077 & $\begin{array}{l}\text { (c) 2019 The Author(s). Published by S. Karger AG, Basel } \\
\text { www.karger.com/cee }\end{array}$ \\
\hline
\end{tabular}

Fisher and Demel: NSAIDs: A Potential Pharmacological Treatment for Intracranial Aneurysm

\section{Introduction}

Saccular intracranial aneurysm (IA) is an outpouching of a cerebral vessel wall, usually occurring at arterial bifurcation sites, with a prevalence of $1-5 \%$ in the general population [1]. Risk factors for IA include hypertension, smoking, female sex, genetic predisposition, and increasing age [2]. While only $20-50 \%$ of IAs rupture, IA rupture leads to subarachnoid hemorrhage (SAH), a devastating type of stroke that has high morbidity and mortality [1]. Half of all SAH survivors suffer neurocognitive deficits that limit their physical and mental abilities, while another third require permanent assistance [3].

Identification of patients with IA has proven difficult. IAs are typically asymptomatic and diagnosed as a result of unrelated imaging. A reliable serum biomarker test for IA detection in the general population has yet to be validated. Even if these IA patients could be identified, there are no nonsurgical interventions to treat IA. While effective, surgical intervention such as clipping and coiling is not always an option; factors such as patient age, genetic background, aneurysm morphology, and surgical risk may make patients ineligible for surgical treatment [4]. A system is in place to determine the risk of rupture. However, the current criteria for weighing rupture risk against surgical risk is based on retrospective analysis and is not accurate. Unfortunately, patients with IA that are not eligible surgical candidates do not have additional treatment options.

In healthy individuals, tissue injury triggers an inflammatory response. This response is characterized by inflammatory cell recruitment, which includes macrophages and lymphocytes. Inflammatory cells produce cytokines and inducible nitric oxide synthase (iNOS). Cytokine activation of iNOS increases nitric oxide (NO) production. In a healthy individual, $\mathrm{NO}$ is an important molecule that regulates vascular tone, maintains blood flow, and regulates mitochondrial oxygen consumption [5]. NO production that supersedes these physiological needs of the body can lead to vascular dysfunction. Patients with IA have a prolonged inflammatory response in which proinflammatory cytokines, such as interleukin-1 (IL-1) and tumor necrosis factor- $\alpha$ (TNF- $\alpha)$, increase the expression of iNOS within vascular smooth muscle cells (VSMCs) and macrophages. Subsequently, there is a large increase in NO production that leads to endothelial cell (EC) and elastin damage, an important structural protein present in vessel walls [6]. Uncoupling of nitric oxide synthase-3 when levels of L-arginine are low can produce superoxide, a reactive oxygen species (ROS) [5]. ROS and inflammation generally occur together and form a positive feedback loop: inflammation leading to neutrophil recruitment, neutrophil production of ROS, and oxidative stress leading to further endothelial injury and more inflammatory cell recruitment.

Nonsteroidal anti-inflammatory drugs (NSAIDs) are currently being investigated as a potential pharmaceutical treatment for patients with IA. Chronic inflammation plays a critical role in IA formation, growth, and eventual rupture. NSAIDs inhibit the cyclooxygenase pathway of arachidonic acid (AA) metabolism, thereby inhibiting platelet-derived thromboxane $\mathrm{A}_{2}\left(\mathrm{TXA}_{2}\right)$ and prostaglandin (PG) synthesis (Fig. 1) [7]. In this review, evidence supporting the use of NSAIDs as a treatment for IA will be presented. First, the pathophysiology of IA formation will be described. Next, evidence will be presented demonstrating that NSAIDs may prevent the further growth of IAs by reducing inflammation, increasing inflammatory cell adhesions to EC, and delaying EC senescence. Last, outcomes of completed clinical trials involving the efficacy of NSAIDs in preventing IA growth will be presented and future planned trials discussed. 


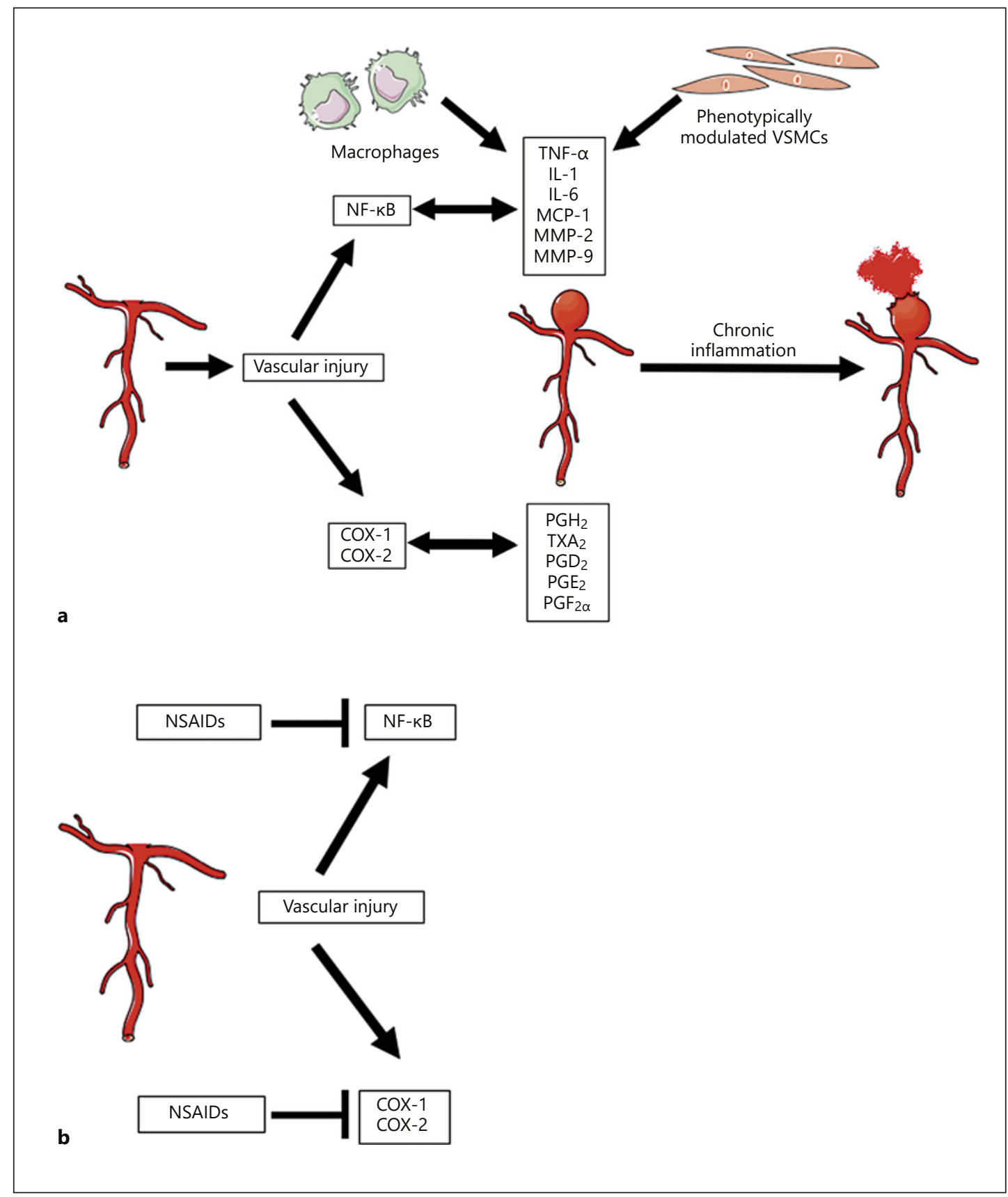

Fig. 1. a Nuclear factor $-\kappa B(N F-\kappa B)$, cyclooxygenase-1 (COX-1), and -2 (COX-2) are activated in response to vascular injury. NF- $\kappa \mathrm{B}$ can directly induce gene expression of proinflammatory cytokines tumor necrosis factor- $\alpha$ (TNF- $\alpha$ ), interleukin-1 (IL-1), interleukin-6 (IL-6), monocyte chemoattractant protein-1 (MCP-1), and matrix metalloproteinase-2 (MMP-2), and -9 (MMP-9). Activation of COX-1 and COX-2 results in increased production of prostanoids prostaglandin $\mathrm{H}_{2}\left(\mathrm{PGH}_{2}\right)$, prostaglandin $\mathrm{D}_{2}\left(\mathrm{PGD}_{2}\right)$, prostaglandin $\mathrm{E}_{2}$ $\left(\mathrm{PGE}_{2}\right)$, prostaglandin $\mathrm{F}_{2 \alpha}\left(\mathrm{PGF}_{2 \alpha}\right)$, and thromboxane $\mathrm{A}_{2}\left(\mathrm{TXA}_{2}\right)$. A positive feedback loop of inflammation forms between vascular smooth muscle cells (VSMCs), macrophages, and proinflammatory cytokines, as well as COX enzymes and prostanoids, eventually leading to IA rupture. $\mathbf{b}$ Inhibition of NF- $\kappa \mathrm{B}, \mathrm{COX}-1$, and COX-2 by nonsteroidal anti-inflammatory drugs (NSAIDs) blocks the production of proinflammatory cytokines, matrix metalloproteinases, and prostaglandins. Reduced production of proinflammatory cytokines may prevent IA rupture $[7,60]$. 


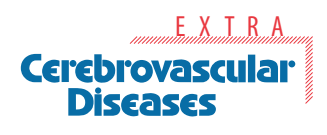

\begin{tabular}{l|l}
\hline Cerebrovasc Dis Extra 2019;9:31-45 \\
\hline DOI: 10.1159/000499077 & $\begin{array}{l}\text { (c) 2019 The Author(s). Published by S. Karger AG, Basel } \\
\text { www.karger.com/cee }\end{array}$ \\
\hline
\end{tabular}

Fisher and Demel: NSAIDs: A Potential Pharmacological Treatment for Intracranial Aneurysm

\section{Inflammation and IA Pathophysiology}

IA is a disorder that is initiated and sustained through a complex and chronic inflammatory response. The most common types of IA are saccular, or berry, aneurysms. These aneurysms represent between 70 and $80 \%$ of all IAs [8]. Histologically, saccular aneurysms have dysfunctional ECs, degraded or no internal elastic lamina, and disorganized smooth muscle cells (SMC) [9]. Hypertension is one of the leading modifiable risk factors for IA. Hemodynamic changes in blood flow result in increased vessel wall sheer stress which commonly occurs at arterial branch points within the circle of Willis. Degradation of the internal elastic lamina, which can occur as a result of hypertension, precedes IA formation [10]. Injury to the endothelium from increased sheer stress results in macrophage recruitment and tissue infiltration. Macrophage infiltration plays a key role in IA formation, as evidenced by macrophage depletion reducing IA prevalence in mice [10]. Continued vascular insult and inflammation leads to breakdown of the internal elastic lamina and loss of structural strength within the arterial wall. Eventually, with continued insult and prolonged activation of self-amplifying inflammatory pathways, IA rupture can occur.

Following vascular injury, PGs are released and act as mediators of the acute inflammatory process. PGs are ubiquitously produced lipids that mediate autocrine and paracrine signaling throughout the body [11]. In the case of IA, we are interested in the PGs and PG receptors that play a role in regulating this inflammatory response. Prostaglandin $\mathrm{E}_{2}\left(\mathrm{PGE}_{2}\right)$ is the most abundant prostanoid in the body and has long been a target for treating inflammation in a variety of diseases [12]. Upon injury to the endothelium, AA is released from phospholipids within the nuclear membrane through the actions of cytosolic phospholipase $A_{2 \alpha}$ $\left(\mathrm{cPLA}_{2 \alpha}\right)$. COX-1 and/or -2 oxygenates AA into prostaglandin $\mathrm{G}_{2}$ which is then enzymatically reduced into prostaglandin $\mathrm{H}_{2}\left(\mathrm{PGH}_{2}\right)$ [12]. $\mathrm{PGH}_{2}$ is relatively unstable and is quickly converted into one of five prostanoids: $\mathrm{PGE}_{2}$, prostaglandin $\mathrm{I}_{2}\left(\mathrm{PGI}_{2}\right)$, prostaglandin $\mathrm{D}_{2}\left(\mathrm{PGD}_{2}\right)$, prostaglandin $\mathrm{F}_{2 \alpha}\left(\mathrm{PGF}_{2 \alpha}\right)$, or $\mathrm{TXA}_{2}$ [12]. $\mathrm{PGE}_{2}$ is synthesized through $\mathrm{PGH}_{2}$ isomerization by microsomal prostaglandin E synthase-1 (mPGES-1) or cytosolic prostaglandin E synthase (cPGES) [13]. Once released from the cell by diffusion, or through its transporter multidrug resistanceassociated protein 4 (MRP4), $\mathrm{PGE}_{2}$ can go and bind to its receptor, prostaglandin $\mathrm{E}_{2}$ receptor $2\left(\mathrm{EP}_{2}\right)$ (Fig. 2).

In response to $\mathrm{EC}$ damage, $\mathrm{PGE}_{2}-\mathrm{EP}_{2}$ signaling is triggered and a positive feedback loop between $\mathrm{EP}_{2}$, nuclear factor- $\kappa \mathrm{B}(\mathrm{NF}-\kappa \mathrm{B})$, and COX-2 is initiated (Fig. 2) [14]. NF- $\kappa \mathrm{B}$ signaling is activated in response to prolonged stress to the endothelium and is accompanied by dysregulation of anti-inflammatory pathways [14]. NF- $\kappa B$ activation upregulates the expression of monocyte chemoattractant protein 1 (MCP-1), a protein which recruits macrophages. NF- $\mathrm{B}$ activation is also implicated in the regulation of VSMC apoptosis [10, 15]. IA initiation and growth are driven by a self-perpetuating cycle of inflammation. While COX-1 can metabolize AA into inflammatory PGs, it is not involved in the positive feedback loops associated with chronic inflammation as is COX-2 (Fig. 2).

Increased mechanical stress to the endothelium, as a result of increased blood flow, causes deterioration of EC tight junctions allowing macrophages to migrate into the vessel wall [16]. Infiltrating macrophages then release proinflammatory cytokines such as TNF- $\alpha$, IL-1, and interleukin-6 (IL-6), resulting in cytokine release from the infiltrating macrophages and further macrophage recruitment and amplification of the inflammatory response [17]. In addition, TNF- $\alpha$ and NO released by the macrophages can also directly activate the NF- $\kappa \mathrm{B}$ pathway (Fig. 3) [6,18]. iNOS increases NO levels leading to the generation of ROS and subsequent oxidative stress in animal and human aneurysm models [6]. In addition to cytokines, macrophages release matrix metalloproteinases (MMPs) that degrade the extracellular matrix (ECM) and are responsible, in part, for vascular remodeling [10]. Infiltration of inflam- 


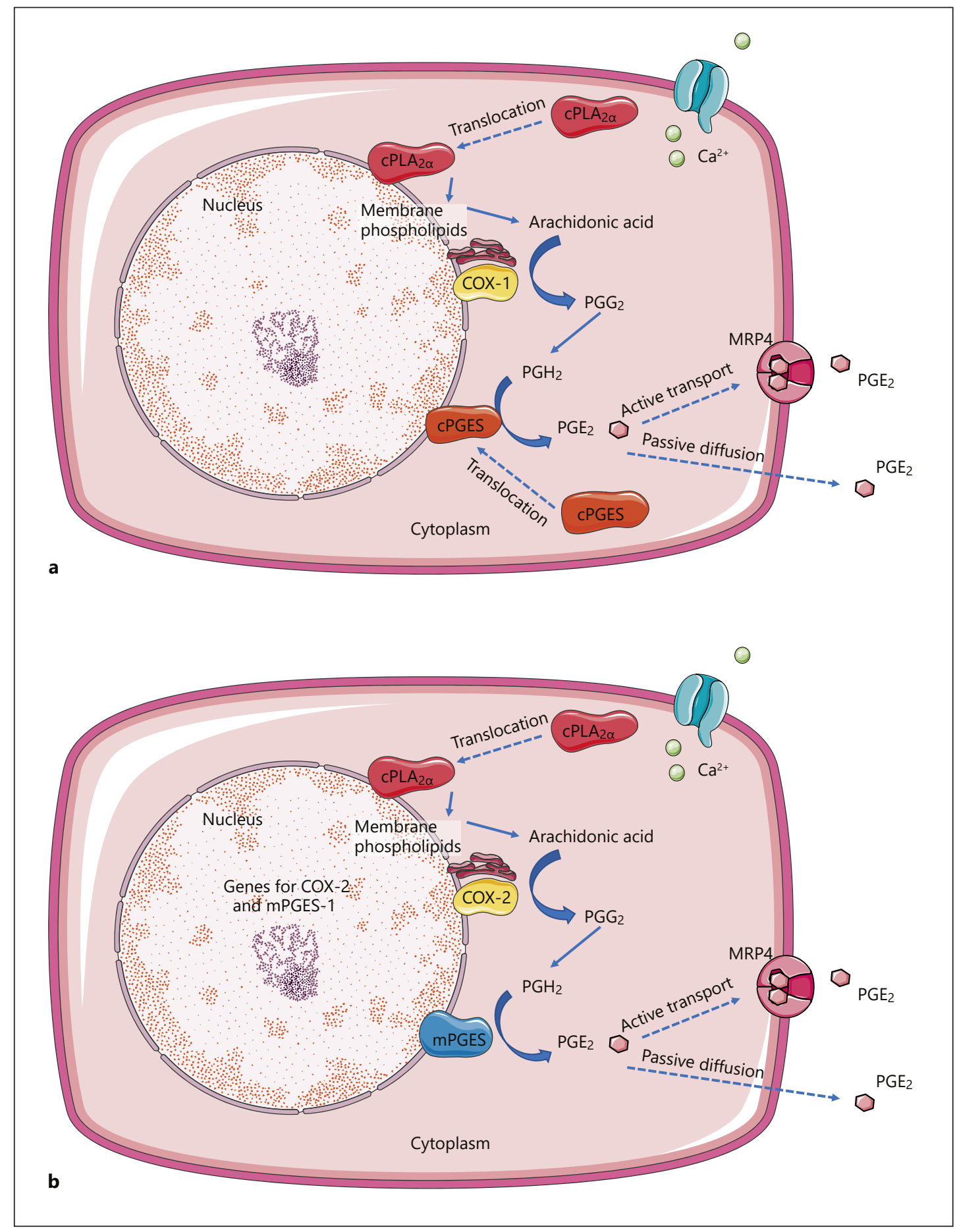

Fig. 2. a Cyclooxygenase-1 (COX-1) is constitutively expressed. Vascular insult causes an inflammatory response resulting in $\mathrm{Ca}^{2+}$ influx into an endothelial cell, triggering translocation of cytoplasmic phospholipase $\mathrm{A}_{2 \alpha}\left(\mathrm{CPLA}_{2}\right)$ to the nucleus. Arachidonic acid is then released from the nuclear membrane and oxygenated by COX-1 producing prostaglandin $\mathrm{G}_{2}\left(\mathrm{PGG}_{2}\right)$, which is quickly enzymatically reduced into prostaglandin $\mathrm{H}_{2}$ $\left(\mathrm{PGH}_{2}\right)$. Cytoplasmic prostaglandin E synthase (cPGES) translocates to the nucleus where it works to convert $\mathrm{PGH}_{2}$ to prostaglandin $\mathrm{E}_{2}\left(\mathrm{PGE}_{2}\right)$. b Upon inflammatory stimuli, there is increased transcription and expression on the nuclear membrane of cyclooxygenase-2 (COX-2) and microsomal prostaglandin E synthase 1 (mPGES-1). mPGES-1, instead of cPGES, converts $\mathrm{PGH}_{2}$ into $\mathrm{PGE}_{2}$ where it can diffuse out of the cell or be actively transported through its transporter, multidrug resistance-associated protein 4 (MRP4) [12, 60]. 


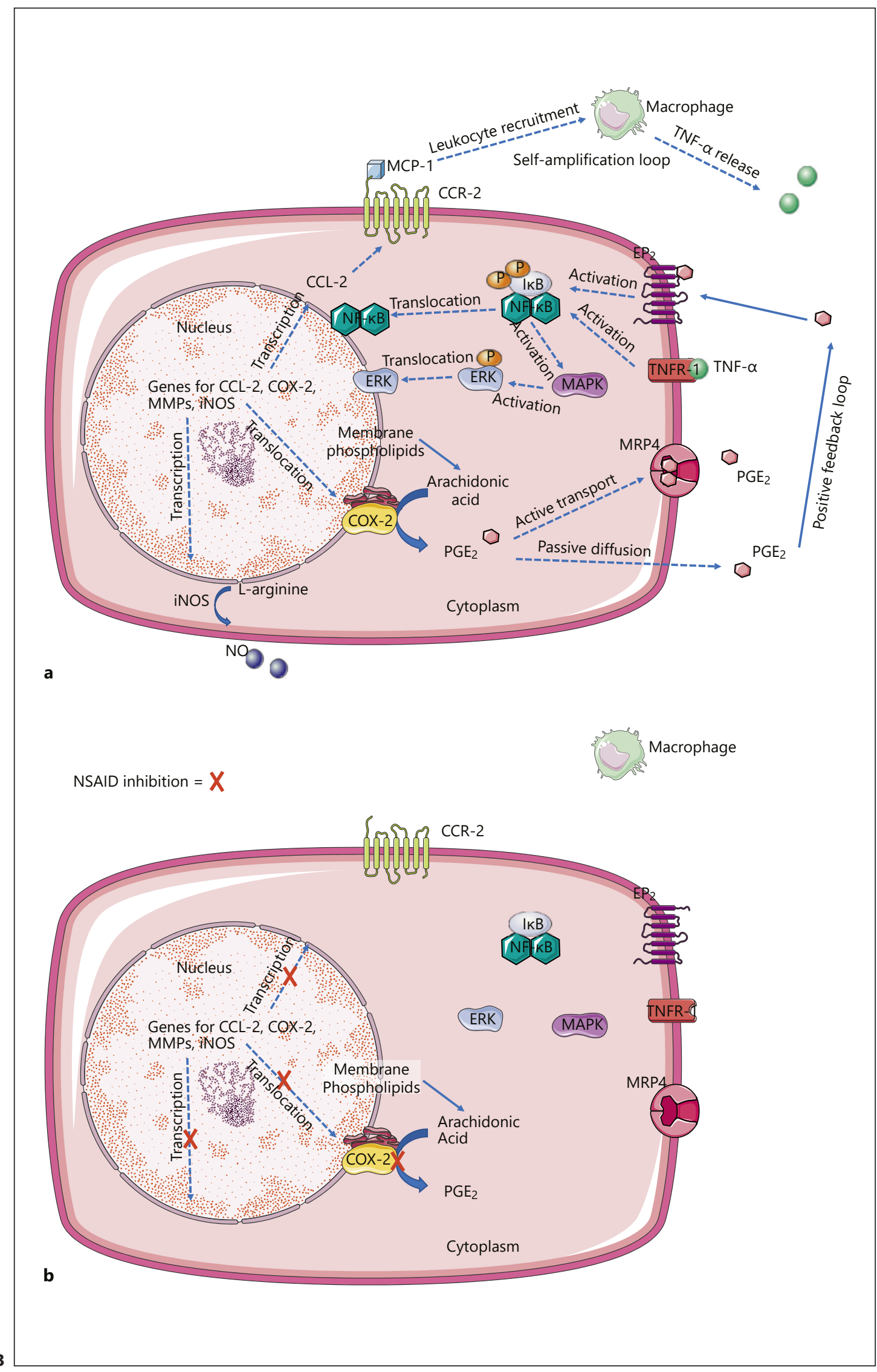


Fisher and Demel: NSAIDs: A Potential Pharmacological Treatment for Intracranial Aneurysm

matory cells induces SMC proliferation and phenotypic changes that result in chronic remodeling of the vascular wall [19]. Attenuating inflammation in the early stages of IA may result in the stabilization of IAs and prevent future rupture by decreasing activation of MMPs and subsequent ECM degradation.

\section{General Overview and Actions of NSAIDs on COX Enzymes}

NSAIDs have anti-inflammatory, anti-pyretic, and analgesic properties making them one of the most widely prescribed class of drugs. This class of drugs elicit their effects by acting on COX-1 and -2 enzymes, the rate-limiting step of AA metabolism. NSAIDs can be placed into one of two groups, nonselective COX inhibitors and selective COX-2 inhibitors. Nonselective COX inhibitors can either reversibly inhibit the actions of both COX-1 and COX-2 enzymes or irreversibly inhibit these actions, as is the case with acetylsalicylic acid (aspirin). Selective COX-2 inhibitors reversibly inhibit enzymatic activity of only COX-2. COX-1 inhibition is associated with the negative side effects typically associated with NSAID use. Inhibition of COX-1 can lead to gastrointestinal toxicity and cardiovascular side effects including stomach ulcers, increased bleeding risk, heart attack, and stroke [20]. COX-1 activity converts AA into $\mathrm{PGI}_{2}$ which decreases parietal cell secretion of $\mathrm{H}^{+}$within the stomach mucosa. As a result, $\mathrm{HCO}_{3}{ }^{-}$ and mucus secretion increase; both of which protect the lining of the stomach. $\mathrm{TXA}_{2}$, which is also produced by COX-1, increases platelet aggregation. Inhibition of COX-1 by NSAIDs can cause acidosis of the stomach and reduced platelet aggregation leading to the cardiovascular and gastrointestinal side effects previously mentioned.

The structures of COX-1 and COX-2 are fairly similar. Both enzymes contain three main domains: an N-terminal epidermal growth factor domain, a helical membrane binding domain (MBD), and a catalytic domain on the C-terminus [12]. NSAIDs and AA both enter into the COX active site through four helices within the MBD and then bind to the active site on the C-terminus [12]. The C-terminal COX-1 active site contains a leucine, while the COX-2 active site contains valine [21]. This small difference in the active sites of COX-1 and COX-2 makes it possible for specific pharmacological inhibition of COX-2.

Selective COX-2 inhibitors were developed in an effort to reduce the serious side effects associated with COX-1 inhibition while still garnering the anti-inflammatory effects. Unlike other NSAIDs, aspirin irreversibly acetylates the serine residue within the active site of COX-1 and modifies enzymatic activity of COX-2 such that COX-2 produces anti-inflammatory lipoxins [22]. In order for COX-1 activity to increase, new enzymes must be synthesized. While

Fig. 3. a Increased hemodynamic stress initiates the recruitment of macrophages, which release the cytokine tumor necrosis factor- $\alpha$ (TNF- $\alpha$ ). TNF- $\alpha$ binds to tumor necrosis factor receptor 1 (TNFR1), while prostaglandin $\mathrm{E}_{2}$ binds to prostaglandin $\mathrm{E}_{2}$ receptor $2\left(\mathrm{EP}_{2}\right)$. Once bound, TNFR1 and $\mathrm{EP}_{2}$ activate nuclear factor- $\mathrm{KB}$ $(\mathrm{NF}-\kappa \mathrm{B})$, causing it to translocate to the nucleus. NF- $\kappa \mathrm{B}$ activation by TNF- $\alpha$ also activates mitogen-activated protein kinase (MAPK), which phosphorylates extracellular signal-receptor kinase (ERK) and subsequently translocates to the nucleus. Once inside the nucleus, NF- $\kappa B$ and ERK increase transcription of various proinflammatory genes including cyclooxygenase-2 (COX-2), chemokine receptor 2 (CCL-2), matrix metalloproteinases (MMPs), and inducible nitric oxide synthase (iNOS). CCL-2 stimulates expression of the C-C chemokine receptor-2 (CCR-2) which, when bound by monocyte chemoattractant protein-1 (MCP-1), recruits leukocytes in a self-amplifying loop. Increased expression of COX-2 leads to increased oxygenation and reduction of arachidonic acid into $\mathrm{PGE}_{2}$ forming a positive feedback loop between EP2-NF- $\mathrm{BB}-\mathrm{COX}-2$. iNOS is converted by L-arginine into nitric oxide (NO), a reactive oxygen species. b Treatment with nonsteroidal anti-inflammatory drugs (NSAIDs) prevents COX-2 conversion of arachidonic acid and all downstream $\mathrm{PGE}_{2}$ signaling effects $[12,17,25,34,60]$. 
Fisher and Demel: NSAIDs: A Potential Pharmacological Treatment for Intracranial Aneurysm

Table 1. Subtype, preferential selectivity of cyclooxygenase (COX) inhibition, actions on COX enzymes, plasma half-life, and cardiovascular (CV) and/or gastrointestinal (GI) system side effect classifications of various nonsteroidal anti-inflammatory drugs (NSAIDs) discussed in this review [20, 28, 30, 47, 48]

\begin{tabular}{llllll}
\hline NSAID & Subtype & $\begin{array}{l}\text { Preferential } \\
\text { selectivity }\end{array}$ & Inhibition of COX & Half-life, h & $\begin{array}{l}\text { Side } \\
\text { effects }\end{array}$ \\
\hline Aspirin & Nonselective COX inhibitor & COX-1 & Irreversible & $\begin{array}{l}\text { Low dose: 2-3 } \\
\text { High dose: 15-30 }\end{array}$ & CV, GI \\
Indomethacin & Nonselective COX inhibitor & COX-2 & Reversible & 4.5 & CV, GI \\
$\begin{array}{l}\text { Piroxicam } \\
\text { Celecoxib }\end{array}$ & Nonselective COX inhibitor & COX-2 & Reversible & 50 & CV, GI \\
Rofecoxib & Selective COX-2 inhibitor & COX-2 & Reversible & 11 & CV \\
\hline
\end{tabular}

this effect on COX-2 is desirable in mediating the inflammatory response, a single dose of aspirin can inhibit platelet aggregation for several days leading to undesirable cardiovascular side effects [23]. However, drugs from both of these NSAID subgroups including aspirin, have been investigated as potential therapeutics for the treatment of IA with varying degrees of success. The majority of studies conducted aiming to assess the effectiveness of NSAIDs for the treatment of IA have been done on five specific NSAIDS: aspirin, indomethacin, piroxicam, celcoxib, and rofecoxib (Table 1).

\section{Evidence that NSAIDs Decrease PGs and ROS in IA}

\section{NSAIDs Reduce Inflammation in IA via Inhibition of COX-2 and PGs}

Infiltration of inflammatory cells into the vessel wall is a universal characteristic of both ruptured and unruptured IA. There are a greater number of inflammatory cells present in ruptured IAs compared to unruptured, suggesting that inflammation plays a role in the growth and destabilization of IAs [2]. In fact, leukocyte infiltration positively correlates with degradation of the ECM and loss of SMCs in the vessel wall [24]. In addition, both COX-2 and the $\mathrm{PGE}_{2}$ receptor $\mathrm{EP}_{2}$ are upregulated in $\mathrm{ECs}$ from ruptured human IAs compared to unruptured IAs [14].

Inflammation in IA is initiated and maintained through a positive feedback loop maintained by the COX-2-PGE $2-\mathrm{EP}_{2}-\mathrm{NF}-\kappa \mathrm{B}$ signaling pathway [14]. In response to vascular insult, COX-2 is induced leading to increased production of $\mathrm{PGE}_{2} . \mathrm{PGE}_{2}$ binds the $\mathrm{EP}_{2}$ receptor which in turn activates NF- $\mathrm{\kappa B}$, an important mediator of proinflammatory cytokine activation [14]. $\mathrm{NF}-\kappa \mathrm{B}$ is a transcription factor that is an important regulator of the inflammatory response and its activity is mediated by a host of intricate signaling cascades. Twenty-seven cytokines and chemokines are under the direct control of NF- $\kappa B$. TNF- $\alpha$ directly activates NF- $\kappa B$ and COX-2 in a positive feedback loop (Fig. 3) [25]. While these actions are important during an acute inflammatory response, prolonged induction of COX-2, MMPs, and other proinflammatory cytokines drive IA progression and may lead to eventual IA rupture.

In a mouse model of IA, EC-activated NF- $\mathrm{KB}$ activity was suppressed with the administration of the selective COX-2 inhibitor celecoxib [14]. Treatment of rats that had undergone IA induction with celecoxib for a period of 12 weeks showed a decreased incidence of IA [14]. It is noteworthy that treatment within the first 6 weeks was as effective as treatment for 12 weeks, suggesting that COX-2 inhibition in the early phase of IA development is important. However, the dose of celecoxib that was used in these studies ( $150 \mathrm{mg} / \mathrm{kg} / \mathrm{day}$ ) is 2.5 times 
higher than the highest recommend daily dose for humans (200 mg/day) [26]. NF- $\kappa B$ p50 subunit deficiency prevented NF- $\kappa B$ from translocating from the cytosol to the nucleus, effectively preventing downstream gene transcription in response to endothelial injury. NF- $\mathrm{B}$ p50 subunit deficient mice had decreased IA size and internal elastic lamina damage [27]. Complete blockade of NF- $\mathrm{KB}$, through the use of a decoy oligodeoxynucleotide which produces anti-NF- $\kappa B$ effects, reduced macrophage infiltration into IA walls and prevented formation of IA entirely in rats [27]. In an elastase model of abdominal aortic aneurysm (AAA) in rats, treatment with the nonselective COX inhibitor indomethacin reduced aortic dilation associated with elastase infusion and prevented the rapid increase in $\mathrm{PGE}_{2}$ synthesis that was seen in the elastase group [28]. Expanding on the previous findings, treatment of rats with indomethacin attenuated the loss of elastin and expression of MMP-9 mRNA when compared to vehicle-treated controls [6]. Taken together, treatment of rats with NSAIDs belonging to the nonselective COX and selective COX-2 subgroups effectively decreased IA growth which likely occurred through inhibition of the $\mathrm{COX}-2-\mathrm{PGE}_{2}-\mathrm{EP}_{2}-\mathrm{NF}-\kappa \mathrm{B}$ signaling pathway.

\section{NSAIDs Reduce ROS in IA via TNF- $\alpha$ Inhibition}

ROS are natural byproducts of cellular metabolism, but when their production surpasses the capacity of the bodies' antioxidant systems, cellular damage can result. In the case of IA, generation of ROS can lead to increased MMP activity and VSMC apoptosis. Aspirin, a nonselective COX inhibitor, inhibits the NF- $\kappa B$ signaling pathway, decreases macrophage activation, and decreases the release of the proinflammatory cytokines IL-6 and TNF- $\alpha$ (Fig. 3) [17]. Treatment of rodents with the TNF- $\alpha$ inhibitor etanercept slows IA formation which is associated with reduced NF- $\mathrm{\kappa B}$ activity and subsequent inhibition of iNOS and MMP expression [18].

NF- $\kappa B$ is controlled by TNF- $\alpha$-tumor necrosis factor receptor 1 (TNF- $\alpha$-TNFR1) signaling, and TNFR1 deficiency leads to reduced IA formation in mice [29]. While TNF- $\alpha$ inhibitors have been shown to be effective in other inflammatory diseases, such as rheumatoid arthritis, they are expensive and have a long duration of action. TNF- $\alpha$ inhibitor effects last for days, resulting in undesired and prolonged immune suppression. Like TNF- $\alpha$ inhibitors, most NSAIDs suppress NF- $\kappa \mathrm{B}$ activation and downstream transcription of proinflammatory genes TNF- $\alpha$, iNOS, and MMPs [25]. However, NSAIDs have a shorter duration of action and are inexpensive compared to TNF- $\alpha$ inhibitors, supporting the idea that NSAIDs are a good therapy candidate for IA [29].

\section{Evidence that NSAIDs Prevent IA Rupture}

\section{NSAIDs and MMPs}

During vascular disease states, MMP expression increases and results in degradation of the ECM and EC tight junctions [16]. There are twenty-five MMPs, of which the gelatinases, MMP-2 and MMP-9, have been implicated in IA and ischemic stroke [30]. Both in vivo animal and clinical studies suggest that NSAIDs, in particular aspirin, inhibit MMPs and prevent IA destabilization and subsequent rupture. Human monocyte-derived macrophages treated with aspirin have reduced MMP-2 and MMP-9 expression and release, as well as reduced expression of NF- $\kappa \mathrm{B}$ [31]. Marfan syndrome, a genetic condition caused by mutations of the fibrillin-1 (FBN-1) gene, often results in the formation of aneurysms with the same characteristics as IA: deterioration of the ECM and chronic inflammation in the vessel wall. Treatment of FBN-1 knockout mice, a relevant model of IA, with the NSAID indomethacin prevents elastin degradation and macrophage infiltration and dampens the upregulation of MMP-2 and MMP-9 expression that is seen in untreated $\mathrm{FBN}-1^{-/-}$mice [32]. In response to inflam- 
Fisher and Demel: NSAIDs: A Potential Pharmacological Treatment for Intracranial Aneurysm

matory stimuli, TNF- $\alpha$ can activate NF- $\mathrm{KB}$ which can bind to MMP-9 and promote expression directly $[33,34]$. TNF- $\alpha$ can also activate mitogen-activated protein kinases, which subsequently phosphorylate extracellular signal-regulated kinase (ERK), resulting in translocation to the nucleus and increased MMP gene transcription (Fig. 3) [34]. Furthermore, treatment of cultured mouse macrophages with aspirin reduced TNF- $\alpha$-induced NF- $\kappa B$ activation and ERK phosphorylation, resulting in decreased MMP-2 and MMP-9 expression [34]. Based on these observations, it is possible that both TNF- $\alpha-\mathrm{NF}-\kappa \mathrm{B}$ and TNF- $\alpha$-ERK signaling work synergistically to influence MMP expression, and that inhibition of MMPs may be due to NSAIDs action on these pathways.

In silico experiments have been conducted to assess the potential effectiveness of the NSAID and nonselective COX inhibitor piroxicam at inhibiting MMPs [30]. The 3-D structures of MMP-2 and MMP-9 receptors and previous pharmacophore information for reference NSAIDs and MMP inhibitors were used to determine how piroxicam would interact with the given molecules in vivo. The active sites of MMP-2 and MMP-9 were investigated and key residues important for MMP enzymatic inhibition were identified, including alanine 84 and leucine 83 on MMP-2 and proline 421 on MMP-9 [30]. Modeling suggests that piroxicam forms stronger hydrogen bonds to the identified critical residues on MMP-2 and MMP-9 compared to other MMP-inhibitors, melatonin and doxycycline. Stronger binding affinity by piroxicam to MMP active sites suggests that it would provide greater inhibition of MMP activity and better prevent the breakdown of the ECM than MMP inhibitors.

\section{NSAIDs and Endothelial Progenitor Cells}

An early feature of IA is dysfunction and degradation of ECs, as evidenced by functional and morphological changes [35]. EC health and function relies upon a balance between the degree of endothelial damage and the body's repair capacity. Injury to the vascular wall stimulates the release of endothelial progenitor cells (EPCs), which are a subpopulation of hematopoietic stem cells that have the ability to proliferate, migrate, and differentiate into ECs thus playing a critical role in vascular repair [36, 37]. Patients at risk for vascular disease have fewer circulating EPCs, decreased EPC migration, and increased senescence compared to healthy controls, suggesting that these patients have impaired ability to repair vessel wall damage $[35,38]$.

NSAIDs affect EPC migration, adhesion, and senescence. EPCs harvested from healthy patients and treated with low-dose aspirin (between 0.1 and $100 \mu \mathrm{mol} / \mathrm{L}$ ) have increased EPC migration, adhesion, and delayed EC senescence but not proliferation [33]. In the same study, high-dose aspirin ( $>1 \mathrm{mmol} / \mathrm{L}$ ) abolished these beneficial effects, suggesting that the effects of aspirin are dose-dependent [33]. This dose dependence has been observed by other investigators $[33,37]$. Aspirin preferentially inhibits COX-1 over COX-2 and begins to inhibit platelet aggregation at low doses. However, COX-2 inhibition requires much higher doses, thereby reducing the positive effects on EPCs. This dose-dependent effect of aspirin on EPCs is important because EPC migration to areas of the vessel wall and increased EPC adhesion may improve a patient's ability to respond to hemodynamic stress. Increased ability for tissue repair may prevent IA growth. However, in the case of aspirin, the dose at which beneficial EPC effects are seen may not be high enough to produce the anti-inflammatory effects of COX-2 inhibition.

\section{NSAIDs and VSMCs}

VSMCs exist in several different phenotypes. The most common phenotype of VSMCs is the contractile phenotype. Contractile VSMCs act to control blood flow and maintain pressure within vessels. Phenotypic modulation of VSMCs can occur in response to a range of stimuli, resulting in VSMCs changing from a contractile phenotype to a proinflammatory and matrix 
remodeling phenotype [19]. Previous research has suggested that VSMC phenotypic modulation in response to inflammatory stimuli plays a major role in the development of atherosclerosis and IA. The modulation is mediated, in part, by TNF- $\alpha[39,40]$. Animal studies of VSMCs support this idea. VSMCs isolated from a mouse model of AAA have reduced levels of contractile phenotypic markers $\alpha$-actin, desmin, and SM22 $\alpha$ [39]. COX-2 mRNA expression in VSMCs was upregulated in both rodent and human AAA and IA studies [13, 39, 41]. Treatment of AAA mice with the selective COX-2 inhibitor celecoxib significantly decreased VSMC marker expression for differentiation [39]. This decrease in phenotypic modulation in VSMCs can be attributed to a reduction in inflammation as a result of selective COX-2 inhibition, potentially through reduced TNF- $\alpha$ activation of NF- $\kappa B$.

Normally, VSMCs do not produce iNOS, but in response to inflammatory cytokines iNOS production can be induced. Several studies evaluating aspirin and indomethacin have assessed the effects of these NSAIDs on iNOS production in VSMCs. One study evaluated the effects of aspirin on iNOS in primary VSMCs isolated from rat aorta. Aspirin inhibited iNOS protein expression in VSMCs through direct binding to the catalytic domain of iNOS protein preventing its conversion by L-arginine into NO [42]. This direct effect was not seen with indomethacin treatment. However, indomethacin and aspirin inhibit iNOS production indirectly through inhibition of NF- $\kappa B$ activation [43]. Structural differences between indomethacin and aspirin may be responsible for this differential effect on iNOS production.

\section{NSAIDs and Clinical Trials}

Several large clinical trials have been conducted to investigate the efficacy of NSAIDs in the treatment of vascular diseases ranging from peripheral artery disease to IA. While trials involving NSAIDs and IA are the most relevant, trials looking at various other vascular diseases are not without merit. The International Study of Unruptured Intracranial Aneurysms (ISUIA) is the largest case-control study to date investigating aspirin in the treatment of IA [44]. Macrophage infiltration into the vessel wall is thought to be a driving factor in IA pathogenesis and growth. Using the contrast agent ferumoxytol, investigators were able to acquire MRI images of patients with unruptured IAs and quantify macrophage accumulation in the aneurysm wall. Patients that took aspirin at least three times per week over the course of 3 months had a significantly lower risk of IA rupture than patients who never took aspirin, as evidenced by a reduction in ferumoxytol-enhanced MRI signal [45]. It is important to note that the patients enrolled in the ISUIA study had IAs that were classified as having a low-risk of rupture and that IA morphology may affect clinical outcomes of aspirin treatment $[44,46]$.

\section{Selective COX-2 Inhibitor Safety Concerns}

The safety profile of selective COX-2 inhibitors has been brought into question given the increased incidence of serious cardiovascular events attributed to these drugs [47]. Selective COX-2 inhibitors reduce the production of $\mathrm{PGI}_{2}$ and its atheroprotective effects. Concurrently, COX-1 produces $\mathrm{TXA}_{2}$, which stimulates platelet aggregation and vasoconstriction [48]. The combination of a lack of COX-2 and continued COX-1 activity can result in increased heart attack and stroke risk. The Adenomatous Polyp Prevention on Vioxx (APPROVe) trial assessed the safety of Vioxx (rofecoxib), a selective COX-2 inhibitor, and saw increased cardiovascular risks that led Merck and Pfizer to withdraw similar selective COX-2 inhibitors from the market [48]. The Safety in Idiopathic Arthritis: NSAIDs and Celebrex Evaluation Registry (SINCERE) trial did not see any significant effect of celecoxib on cardiovascular thromboembolic events [47]. At high doses, nonselective COX inhibitors have been known to increase incidence of cardiovascular events at a similar rate to those seen with selective COX-2 inhibitors, suggesting 
that safety is dependent upon dose and duration of treatment [48]. A large cohort study showed that administration of the selective COX-2 inhibitors rofecoxib and celecoxib in concert with low-dose aspirin (up to $81 \mathrm{mg} /$ day) reduced the incidence of cardiovascular events typically seen with selective COX-2 inhibitor use alone and reduced the gastrointestinal effects seen with aspirin use alone [49]. Low-dose aspirin, when used concomitantly with selective COX-2 inhibitors, may inhibit COX-1 production of $\mathrm{TXA}_{2}$ enough to reduce platelet aggregation without significantly inhibiting $\mathrm{PGI}_{2}$ and downstream parietal cell function [49]. Specific IA rupture risk, as well as patient cardiovascular medical history and current health status, needs to be weighed against the potential benefits for treatment with either nonselective COX or selective COX-2 inhibitor treatment.

\section{Statins and IA Risk Reduction}

In addition to NSAIDs, other agents have shown promise in reducing IA development and rupture. Of these agents, statins, known as 3-hydroxy-3-methylglutaryl coenzyme A (HMG$\mathrm{CoA}$ ) reductase inhibitors, have been shown to have anti-inflammatory properties that are independent of their lipid-lowering effect [50-52]. However, the effect of statins on aneurysm growth is a disputed topic.

In a clinical study of aorta from patients on statin therapy for at least 6 weeks prior to abdominal aortic, aneurysm repair found that statins reduced the expression of selected inflammatory mediators within the vessel wall through a shift in the M1/M2 balance, and reduced markers linked to macrophage differentiation [53]. However, in this investigation, statins did not reduce aortic aneurysm growth, making the clinical relevance in this setting unclear. A recent systematic review and meta-analysis of statin use and AAA growth, rupture, and perioperative mortality found that statins were associated with a lower risk of all three [54].

That being said, the pathological processes underlying abdominal aneurysms and cerebral aneurysms are different, with less focus on atherosclerotic disease, and more on hemodynamic and genetic factors. Furthermore, the low incidence rate has made proving the effectiveness of statins on aneurysm rupture difficult. Multiple animal model studies have demonstrated reduced aneurysm rupture [55-57]. Additionally, smaller case-control studies in specific populations have shown reduced incidence of cerebral aneurysm rupture in patients taking statins; however, this study had several limitations based on its design and inequalities between groups [58]. When associations between aneurysm formation and statins were addressed in another case-control study, no association was found [59].

Further research is needed to assess the role of statins in cerebral aneurysm formation, growth and rupture risk. A better delineation of the nonlipid mechanisms of anti-inflammatory effects is warranted along with prospective clinical studies before further recommendations can be made.

\section{Conclusions}

IA is a chronic inflammatory disease of the arterial wall characterized by EC degradation and VSMC dysfunction. NSAIDs are a promising drug therapy for IA and prevention of SAH. These drugs decrease the chronic inflammation characteristic of IA through the inhibition of the $\mathrm{COX}-2-\mathrm{PGE}_{2}-\mathrm{EP}_{2}-\mathrm{NF}-\kappa \mathrm{B}$ signaling pathway and could significantly attenuate IA rupture risk. NSAIDs effectively inhibit COX-2, thereby reducing the activity of NF- $\kappa \mathrm{B}$ and the genes NF- $\kappa B$ induces, including TNF- $\alpha$, iNOS, and MMPs. Aspirin, as well as other nonselective and 
selective COX-2 inhibitors, are known to inhibit the release of proinflammatory cytokines TNF- $\alpha$, MCP-1, IL-1, and IL-6. In addition, TNF- $\alpha$ inhibition in rats by etanercept reduces ROS and MMP production in IA. Long-term use of both subtypes of NSAIDs can lead to serious cardiovascular side effects. Due to these increased risks, studies focusing on specific dosedependent effects and duration of treatment need to be conducted.

Presently, there are two active clinical trials that are aimed at assessing the effectiveness of aspirin in preventing IA rupture. The first trial, The Clinical Benefit and Risk of Oral Aspirin for Patients Who Have Unruptured Intracranial Aneurysm Combined with Cerebral Ischemia (CBROAUNACCI), is being conducted in Beijing. This trial will identify patients with cerebral ischemia and IA that would be suited for aspirin therapy and determine the effects aspirin treatment has on preventing IA rupture. The second trial based in Germany, Acetylsalicylic Acid Plus Intensive Blood Pressure Treatment in Patients with Unruptured Intracranial Aneurysms (PROJECT-U), is assessing a combination therapy of aspirin and any blood pressure treatment on unruptured IA compared to standard care.

Selective COX-2 inhibitors are still a relatively new class of NSAIDs. Current clinical trials are focusing on the nonselective COX inhibitor aspirin, most likely due to the cardiovascular concerns associated with selective COX-2 inhibition. Further research should be conducted on the dose-dependent effects and cardiovascular risks associated with selective COX-2 inhibitors given that they are still a relatively new class of drug. Additional studies should focus on assessing the safety and efficacy of concurrent use of low-dose aspirin and selective COX-2 inhibitors to treat IA. In conclusion, the current basic science and clinical data support NSAID therapy in preventing IA rupture, but more research is needed minimize deleterious cardiovascular and gastrointestinal risks.

\section{Acknowledgements}

The authors would like to acknowledge Dr. Anne Dorrance and Dr. Theresa Lansdell for providing editorial support.

\section{Disclosure Statement}

The authors have nothing to disclose.

\section{References}

1 Ajiboye N, Chalouhi N, Starke RM, Zanaty M, Bell R. Unruptured cerebral aneurysms: evaluation and management. ScientificWorldJournal. 2015;2015:954954.

2 Frösen J, Piippo A, Paetau A, Kangasniemi M, Niemelä M, Hernesniemi J, et al. Remodeling of saccular cerebral artery aneurysm wall is associated with rupture: histological analysis of 24 unruptured and 42 ruptured cases. Stroke. 2004 Oct;35(10):2287-93.

3 Starke RM, Chalouhi N, Ding D, Hasan DM. Potential role of aspirin in the prevention of aneurysmal subarachnoid hemorrhage. Cerebrovasc Dis. 2015;39(5-6):332-42.

4 Aoki T, Saito M, Koseki H, Tsuji K, Tsuji A, Murata K, et al.; MR Macrophage Imaging Study Investigators. Macrophage Imaging of Cerebral Aneurysms with Ferumoxytol: an Exploratory Study in an Animal Model and in Patients. J Stroke Cerebrovasc Dis. 2017 Oct;26(10):2055-64.

5 Luiking YC, Engelen MP, Deutz NE. Regulation of nitric oxide production in health and disease. Curr Opin Clin Nutr Metab Care. 2010 Jan;13(1):97-104.

6 Armstrong PJ, Franklin DP, Carey DJ, Elmore JR. Suppression of experimental aortic aneurysms: comparison of inducible nitric oxide synthase and cyclooxygenase inhibitors. Ann Vasc Surg. 2005 Mar;19(2):248-57. 


\begin{tabular}{l|l}
\hline Cerebrovasc Dis Extra 2019;9:31-45 \\
\hline DOI: 10.1159/000499077 & $\begin{array}{l}\text { (c) 2019 The Author(s). Published by S. Karger AG, Basel } \\
\text { www.karger.com/cee }\end{array}$ \\
\hline
\end{tabular}

Fisher and Demel: NSAIDs: A Potential Pharmacological Treatment for Intracranial Aneurysm

7 Morris T, Stables M, Hobbs A, de Souza P, Colville-Nash P, Warner T, et al. Effects of low-dose aspirin on acute inflammatory responses in humans. J Immunol. 2009 Aug;183(3):2089-96.

8 Spetzler RF, Zabramski JM, McDougall CG, Albuquerque FC, Hills NK, Wallace RC, et al. Analysis of saccular aneurysms in the Barrow Ruptured Aneurysm Trial. J Neurosurg. 2018 Jan;128(1):120-5.

9 Schievink WI. Intracranial aneurysms. N Engl J Med. 1997 Jan;336(1):28-40.

10 Kanematsu Y, Kanematsu M, Kurihara C, Tada Y, Tsou TL, van Rooijen N, et al. Critical roles of macrophages in the formation of intracranial aneurysm. Stroke. 2011 Jan;42(1):173-8.

11 Ricciotti E, FitzGerald GA. Prostaglandins and inflammation. Arterioscler Thromb Vasc Biol. 2011 May;31(5): 986-1000.

12 Park JY, Pillinger MH, Abramson SB. Prostaglandin E2 synthesis and secretion: the role of PGE2 synthases. Clin Immunol. 2006 Jun;119(3):229-40.

13 Hasan D, Hashimoto T, Kung D, Macdonald RL, Winn HR, Heistad D. Upregulation of cyclooxygenase-2 (COX-2) and microsomal prostaglandin E2 synthase-1 (mPGES-1) in wall of ruptured human cerebral aneurysms: preliminary results. Stroke. 2012 Jul;43(7):1964-7.

14 Aoki T, Nishimura M, Matsuoka T, Yamamoto K, Furuyashiki T, Kataoka H, et al. PGE(2) -EP(2) signalling in endothelium is activated by haemodynamic stress and induces cerebral aneurysm through an amplifying loop via NF-кB. Br J Pharmacol. 2011 Jul;163(6):1237-49.

15 Chalouhi N, Atallah E, Jabbour P, Patel PD, Starke RM, Hasan D. Aspirin for the Prevention of Intracranial Aneurysm Rupture. Neurosurgery. 2017 Sep;64 CN_suppl_1:114-8.

16 Tada Y, Yagi K, Kitazato KT, Tamura T, Kinouchi T, Shimada K, et al. Reduction of endothelial tight junction proteins is related to cerebral aneurysm formation in rats. J Hypertens. 2010 Sep;28(9):1883-91.

17 Liu Y, Fang S, Li X, Feng J, Du J, Guo L, et al. Aspirin inhibits LPS-induced macrophage activation via the NF- $\kappa B$ pathway. Sci Rep. 2017 Sep;7(1):11549.

18 Yokoi T, Isono T, Saitoh M, Yoshimura Y, Nozaki K. Suppression of cerebral aneurysm formation in rats by a tumor necrosis factor- $\alpha$ inhibitor. J Neurosurg. 2014 May;120(5):1193-200.

19 Starke RM, Chalouhi N, Ding D, Raper DM, Mckisic MS, Owens GK, et al. Vascular smooth muscle cells in cerebral aneurysm pathogenesis. Transl Stroke Res. 2014 Jun;5(3):338-46.

20 Süleyman H, Demircan B, Karagöz Y. Anti-inflammatory and side effects of cyclooxygenase inhibitors. Pharmacol Rep. 2007 May-Jun;59(3):247-58.

21 Gierse JK, McDonald JJ, Hauser SD, Rangwala SH, Koboldt CM, Seibert K. A single amino acid difference between cyclooxygenase-1 (COX-1) and -2 (COX-2) reverses the selectivity of COX-2 specific inhibitors. J Biol Chem. 1996 Jun;271(26):15810-4.

22 Chen TG, Chen JZ, Xie XD. Effects of aspirin on number, activity and inducible nitric oxide synthase of endothelial progenitor cells from peripheral blood. Acta Pharmacol Sin. 2006 Apr;27(4):430-6.

23 Meek IL, Van de Laar MA, E Vonkeman H. Non-Steroidal Anti-Inflammatory Drugs: An Overview of Cardiovascular Risks. Pharmaceuticals (Basel). 2010 Jul;3(7):2146-62.

24 Kataoka K, Taneda M, Asai T, Kinoshita A, Ito M, Kuroda R. Structural fragility and inflammatory response of ruptured cerebral aneurysms. A comparative study between ruptured and unruptured cerebral aneurysms. Stroke. 1999 Jul;30(7):1396-401.

25 Yamamoto Y, Gaynor RB. Therapeutic potential of inhibition of the NF-kappaB pathway in the treatment of inflammation and cancer. J Clin Invest. 2001 Jan;107(2):135-42.

26 Pfizer Inc. Celebrex [Internet]. FDA 1999 May 1 [cited 2018 Sep 12]. Available from: https://www.accessdata. fda.gov/drugsatfda_docs/label/1998/20998lbl.pdf

27 Aoki T, Shimamura M, Nakagami H, Wakayama K, Moriwaki T, Ishibashi R, et al. NF-kB is a key mediator of cerebral aneurysm formation. Circulation. 2007 Dec;116(24):2830-40.

28 Miralles M, Wester W, Sicard GA, Thompson R, Reilly JM. Indomethacin inhibits expansion of experimental aortic aneurysms via inhibition of the cox2 isoform of cyclooxygenase. J Vasc Surg. 1999 May;29(5):884-92; discussion 892-3.

29 Aoki T, Fukuda M, Nishimura M, Nozaki K, Narumiya S. Critical role of TNF-alpha-TNFR1 signaling in intracranial aneurysm formation. Acta Neuropathol Commun. 2014 Mar;2(1):34.

30 Mazumder MK, Bhattacharya P, Borah A. Inhibition of matrix metalloproteinase- 2 and 9 by Piroxicam confer neuroprotection in cerebral ischemia: an in silico evaluation of the hypothesis. Med Hypotheses. 2014 Dec; 83(6):697-701.

31 Hua Y, Xue J, Sun F, Zhu L, Xie M. Aspirin inhibits MMP-2 and MMP-9 expressions and activities through upregulation of PPARalpha/gamma and TIMP gene expressions in ox-LDL-stimulated macrophages derived from human monocytes. Pharmacology. 2009;83(1):18-25.

32 Guo G, Ott CE, Grünhagen J, Muñoz-García B, Pletschacher A, Kallenbach K, et al. Indomethacin prevents the progression of thoracic aortic aneurysm in marfan syndrome mice. Aorta (Stamford). 2013 Jun; 1(1):5-12.

33 Hu Z, Zhang F, Yang Z, Zhang J, Zhang D, Yang N, et al. Low-dose aspirin promotes endothelial progenitor cell migration and adhesion and prevents senescence. Cell Biol Int. 2008 Jul;32(7):761-8.

34 Zhang P, Wu C, Huang XH, Shen CL, Li L, Zhang W, et al. Aspirin suppresses TNF- $\alpha$-induced MMP-9 expression via NF- $\mathrm{BB}$ and MAPK signaling pathways in RAW264.7 cells. Exp Ther Med. 2017 Dec;14(6):5597-604.

35 Wei H, Mao Q, Liu L, Xu Y, Chen J, Jiang R, et al. Changes and function of circulating endothelial progenitor cells in patients with cerebral aneurysm. J Neurosci Res. 2011 Nov;89(11):1822-8. 
Fisher and Demel: NSAIDs: A Potential Pharmacological Treatment for Intracranial Aneurysm

36 Li S, Wang D, Tian Y, Wei H, Zhou Z, Liu L, et al. Aspirin inhibits degenerative changes of aneurysmal wall in a rat model. Neurochem Res. 2015 Jul;40(7):1537-45.

37 Lou J, Povsic TJ, Allen JD, Adams SD, Myles S, Starr AZ, et al. The effect of aspirin on endothelial progenitor cell biology: preliminary investigation of novel properties. Thromb Res. 2010 Sep;126(3):e175-9.

38 Vasa M, Fichtlscherer S, Aicher A, Adler K, Urbich C, Martin H, et al. Number and migratory activity of circulating endothelial progenitor cells inversely correlate with risk factors for coronary artery disease. Circ Res. 2001 Jul;89(1):E1-7.

39 Mukherjee K, Gitlin JM, Loftin CD. Effectiveness of cyclooxygenase-2 inhibition in limiting abdominal aortic aneurysm progression in mice correlates with a differentiated smooth muscle cell phenotype. J Cardiovasc Pharmacol. 2012 Dec;60(6):520-9.

40 Ali MS, Starke RM, Jabbour PM, Tjoumakaris SI, Gonzalez LF, Rosenwasser RH, et al. TNF- $\alpha$ induces phenotypic modulation in cerebral vascular smooth muscle cells: implications for cerebral aneurysm pathology. J Cereb Blood Flow Metab. 2013 Oct;33(10):1564-73.

41 Chu Y, Wilson K, Gu H, Wegman-Points L, Dooley SA, Pierce GL, et al. Myeloperoxidase is increased in human cerebral aneurysms and increases formation and rupture of cerebral aneurysms in mice. Stroke. 2015 Jun; 46(6):1651-6.

42 Katsuyama K, Shichiri M, Kato H, Imai T, Marumo F, Hirata Y. Differential inhibitory actions by glucocorticoid and aspirin on cytokine-induced nitric oxide production in vascular smooth muscle cells. Endocrinology. 1999 May;140(5):2183-90.

43 Shimpo M, Ikeda U, Maeda Y, Ohya K, Murakami Y, Shimada K. Effects of aspirin-like drugs on nitric oxide synthesis in rat vascular smooth muscle cells. Hypertension. 2000 May;35(5):1085-91.

44 Yoon W. Current update on the randomized controlled trials of intracranial aneurysms. Neurointervention. $2011 \mathrm{Feb} ; 6(1): 1-5$.

45 Hasan DM, Mahaney KB, Brown RD Jr, Meissner I, Piepgras DG, Huston J, et al.; International Study of Unruptured Intracranial Aneurysms Investigators. Aspirin as a promising agent for decreasing incidence of cerebral aneurysm rupture. Stroke. 2011 Nov;42(11):3156-62.

46 Wiebers DO, Whisnant JP, Huston J 3rd, Meissner I, Brown RD Jr, Piepgras DG, et al.; International Study of Unruptured Intracranial Aneurysms Investigators. Unruptured intracranial aneurysms: natural history, clinical outcome, and risks of surgical and endovascular treatment. Lancet. 2003 Jul;362(9378):103-10.

47 Sobel RE, Lovell DJ, Brunner HI, Weiss JE, Morris PW, Gottlieb BS, et al.; Pediatric Rheumatology Collaborative Study Group. Safety of celecoxib and nonselective nonsteroidal anti-inflammatory drugs in juvenile idiopathic arthritis: results of the Phase 4 registry. Pediatr Rheumatol Online J. 2014 Jul;12(1):29.

48 Zarghi A, Arfaei S. Selective COX-2 Inhibitors: A Review of Their Structure-Activity Relationships. Iran J Pharm Res. 2011;10(4):655-83.

49 Strand V. Are COX-2 inhibitors preferable to non-selective non-steroidal anti-inflammatory drugs in patients with risk of cardiovascular events taking low-dose aspirin? Lancet. 2007 Dec;370(9605):2138-51.

50 Jain MK, Ridker PM. Anti-inflammatory effects of statins: clinical evidence and basic mechanisms. Nat Rev Drug Discov. 2005 Dec;4(12):977-87.

51 Verschuren L, Kleemann R, Offerman EH, Szalai AJ, Emeis SJ, Princen HM, et al. Effect of low dose atorvastatin versus diet-induced cholesterol lowering on atherosclerotic lesion progression and inflammation in apolipoprotein E*3-Leiden transgenic mice. Arterioscler Thromb Vasc Biol. 2005 Jan;25(1):161-7.

52 Kleemann R, Princen HM, Emeis JJ, Jukema JW, Fontijn RD, Horrevoets AJ, et al. Rosuvastatin reduces atherosclerosis development beyond and independent of its plasma cholesterol-lowering effect in APOE*3-Leiden transgenic mice: evidence for antiinflammatory effects of rosuvastatin. Circulation. 2003 Sep;108(11):136874.

53 van der Meij E, Koning GG, Vriens PW, Peeters MF, Meijer CA, Kortekaas KE, et al. A clinical evaluation of statin pleiotropy: statins selectively and dose-dependently reduce vascular inflammation. PLoS One. 2013; 8(1):e53882.

54 Salata K, Syed M, Hussain MA, de Mestral C, Greco E, Mamdani M, et al. Statins Reduce Abdominal Aortic Aneurysm Growth, Rupture, and Perioperative Mortality: A Systematic Review and Meta-Analysis. J Am Heart Assoc. 2018 Oct; 7(19):e008657.

55 Aoki T, Kataoka H, Ishibashi R, Nakagami H, Nozaki K, Morishita R, et al. Pitavastatin suppresses formation and progression of cerebral aneurysms through inhibition of the nuclear factor kappaB pathway. Neurosurgery. 2009 Feb;64(2):357-65; discussion 365-6.

56 Aoki T, Kataoka H, Ishibashi R, Nozaki K, Hashimoto N. Simvastatin suppresses the progression of experimentally induced cerebral aneurysms in rats. Stroke. 2008 Apr;39(4):1276-85.

57 Kimura N, Shimizu H, Eldawoody H, Nakayama T, Saito A, Tominaga T, et al. Effect of olmesartan and pravastatin on experimental cerebral aneurysms in rats. Brain Res. 2010 Mar;1322:144-52.

58 Yoshimura Y, Murakami Y, Saitoh M, Yokoi T, Aoki T, Miura K, et al.; SSS Research Group. Statin use and risk of cerebral aneurysm rupture: a hospital-based case-control study in Japan. J Stroke Cerebrovasc Dis. 2014 Feb;23(2):343-8.

59 Marbacher S, Schläppi JA, Fung C, Hüsler J, Beck J, Raabe A. Do statins reduce the risk of aneurysm development? A case-control study. J Neurosurg. 2012 Mar;116(3):638-42.

60 Servier Medical Art: Servier Medical Art [Internet]. SMART -Servier Medical ART [cited 2018 Apr 12]. Available from: https://smart.servier.com 